\title{
Tumores endobronquiales: revisión del abordaje diagnóstico y tratamiento endoscópico
}

\section{Endobronchial tumors: review of the diagnostic approach and endoscopic treatment}

\author{
Miguel Osejo Betancourt ${ }^{1}$ Edgar Sánchez ${ }^{2}$, Alfredo Saavedra ${ }^{3}$, German Diaz \\ Santos $^{4}$ y Ana Milena Callejas ${ }^{5}$
}

\section{Resumen}

Los tumores endobronquiales puros son entidades pocos frecuentes, que presentan un reto para su diagnóstico y abordaje terapéutico. Por su rareza, son poco conocidos entre los médicos de atención primaria y aun entre médicos con más experiencia, por lo que es común que el diagnóstico sea realizado en estadios avanzados de la enfermedad. Además, se cuenta con poca literatura disponible, con una escasez de estudios en poblaciones grandes, con la evidencia reportada comúnmente en reportes de casos o la experiencia de grupos tratantes. La presentación clínica de estos tumores es poco específica y variable de acuerdo con el grado de obstrucción, con un abordaje diagnóstico basado en imágenes especialmente la tomografía de tórax y la broncoscopia, que corresponde al estudio de elección ya que puede ser diagnóstica y terapéutica. El tratamiento de elección es el manejo endoscópico, con diferentes técnicas, sin evidencia de superioridad de una con respecto a otra, por lo que su elección depende de la disponibilidad y la experticia que tenga el neumólogo intervencionista para realizarla.

Palabras clave: neoplasias; broncoscopia; diagnóstico.

\footnotetext{
Abstract

Pure endobronchial tumors are rare entities that present a challenge for their diagnosis and therapeutic approach. Due to their rarity, they are little known among primary care physicians and even among more expert physicians, so it's common that diagnosis is made in advanced stages. Also, there is little available literature, with a paucity in studies with big populations, with evidence published as case reports or the experience of the treating group. The clinical picture is non-specific and variable, according to the degree of
}

${ }^{1}$ Especialista en Medicina Interna y Neumología, Hospital Santa Clara, Bogotá.

${ }^{2}$ Especialista en Medicina Interna y Neumología, Instituto Nacional de Cancerología, Bogotá.

${ }^{3}$ Especialista en Medicina Interna y Neumología, Instituto Nacional de Cancerología, Bogotá.

${ }^{4}$ Especialista en Medicina Interna y Neumología, Instituto Nacional de Cancerología, Bogotá.

${ }^{5}$ Especialista en Medicina Interna y Neumología, Instituto Nacional de Cancerología, Bogotá.

\section{Autor de Correspondencia Miguel Osejo Betancourt, Correo electrónico: mikeosejo2@gmail.com}

Recibido: 05/07/2021

Aceptado: 09/11/2021 
obstruction, with a diagnostic approach based on images, especially chest tomography and bronchoscopy, which is the study of choice since it can be diagnostic and therapeutic. The treatment of choice is endoscopic management, with diferents technichs without evidence of superiority between them o the choice depends on the availability of each one and the expertise of the interventional pulmonologist to perform it.

Keywords: neoplasm, bronchoscopy, diagnosis.

\section{Introducción}

Los tumores endobronquiales puros son entidades poco frecuentes, con diversas características patológicas que presentan un reto para su diagnóstico y abordaje terapéutico (1).

Representan solo el $0.6 \%$ de las neoplasias pulmonares, siendo más frecuentes los malignos primarios. En orden de frecuencia los tres más comunes son los tumores carcinoides, el carcinoma escamocelular y el carcinoma mucoepidermoide; los tumores secundarios son muy raros, siendo lo más frecuentes invasión directa (aproximadamente 30 $\%$ de los casos de cáncer de pulmón se complica con compromiso luminal), antes que diseminación hematógena $(1,2)$. Es muy raro encontrarlos en la infancia, de igual forma lo común es que sean malignos y se debe sospechar cuando no mejora la clínica respiratoria ni la respuesta adecuada a los broncodilatadores (1).

Los tumores benignos generalmente son de origen mesenquimal, de crecimiento lento, se presentan con obstrucción bronquial, pero la clínica es variable; los más frecuentes son los hamartomas y los papilomas (1).

El tratamiento de elección para estos tumores es endoscópico, para el cual se cuenta con diversas opciones en la actualidad, que dependen de la disponibilidad y la experiencia del médico para cada intervención.

Dado que son poco frecuentes, son poco conocidos por los médicos de atención primaria y aun entre médicos especialistas, por lo que suele dificultarse el diagnóstico hasta que se encuentra en estadios avanzados. Se cuenta con poca literatura disponible sobre ellos, principalmente reportes de casos, por lo que el objetivo de esta revisión es describir el abordaje diagnóstico y terapéutico de los tumores endobronquiales, desde una perspectiva actualizada, tomando en cuenta los avances en imágenes diagnósticas y en las opciones de tratamiento endoscópicos.

\section{Metodología}

Para el desarrollo de este artículo se realizó una revisión narrativa de la literatura en la que se utilizaron las bases de datos PubMed, ClinicalKey, ScienceDirect y Google Scholar, incluyendo artículos publicados en idioma inglés y español, entre el $1^{\circ}$ de enero de 2005 y el 30 de junio de 2021. Además, se complementó la búsqueda de literatura relevante a través de la revisión de referencias de los artículos seleccionados por los autores.

Para la búsqueda de la literatura se emplearon los términos $\mathrm{MeSH}$, seleccionando como palabras claves Bronchoscopy (Broncoscopia), Bronchial Neoplasms (Neoplasias bronquiales), Lung Neoplasms (Neoplasias pulmonares), Cryotherapy (Crioterapia) y Stents con combinaciones utilizando como operadores booleanos AND y OR.

\section{Resultados}

En la búsqueda y síntesis de la literatura, se seleccionaron los artículos de acuerdo con las preferencias del subtema a tratar, incluyendo artículos de revisión, guías y protocolos, estudios observacionales y ensayos clínicos, sin embargo, en estos últimos hay ausencia de publicaciones recientes en los datos arrojados por la búsqueda y según la experiencia de los autores.

\section{Diagnóstico de tumores endobronquiales}

Clínica: en general presentan sintomatología inespecífica y variable, con tos, disnea, hemoptisis, sibilancias localizadas, neumonías recurrentes o atelectasias, debido a obstrucción bronquial (1). Pueden también ser asintomáticos, imitar otras neoplasias o provocar síntomas similares a enfermedad pulmonar obstructiva crónica (EPOC) o asma (1). Cuando el tumor está específicamente en el bronquio del lóbulo medio puede causar síndrome del lóbulo medio, que se caracteriza por atelectasia asintomática y se diagnostica generalmente de forma incidental (3) o asociarse al desarrollo de neumonías postobstructivas, también conocidas cono neumonías 
lipoideas por la formación de vacuolas grasas (4).

Entre las pruebas de función pulmonar la reducción de la ventilación voluntaria máxima (MMV) y el flujo espiratorio pico tienen la mayor sensibilidad, sin embargo, ambos estudios son poco utilizados en la práctica clínica en comparación con otras pruebas de función pulmonar (1).

Otras pruebas más comúnmente utilizadas como la espirometría, que permite la medición del volumen espiratorio forzado al primer segundo (VEF1), pero tiene baja sensibilidad dado que se disminuye hasta etapas avanzadas de la enfermedad, cuando la reducción de la luz traqueal es de al menos 10 $\mathrm{mm}$. La forma de la curva de flujo-volumen puede presentar anormalidades en etapas más tempranas y producir todos los patrones de obstrucción de vía área superior (OVAS), siendo la más frecuente la obstrucción variable intratorácica, que se puede observan con aplanamiento del asa espiratoria de la curva $(2,5)$.

Imágenes: la radiografía de tórax puede ser normal o manifestar hallazgos poco específicos como nódulo solitario, atelectasia o consolidación. En comparación, la tomografía axial computarizada (TAC) de tórax es el estudio de elección, ya que permite una mejor visualización de lesiones, evalúa la extensión del tumor y permite explorar diagnósticos diferenciales. Los principales hallazgos tomográficos corresponden a masa bronquial o traqueal (Figura 1), dilatación bronquial distal, impactación de moco, atelectasia segmentaria, neumonía posobstructiva o atrapamiento aéreo y permite observar lesiones parenquimatosas o mediastinales (1).

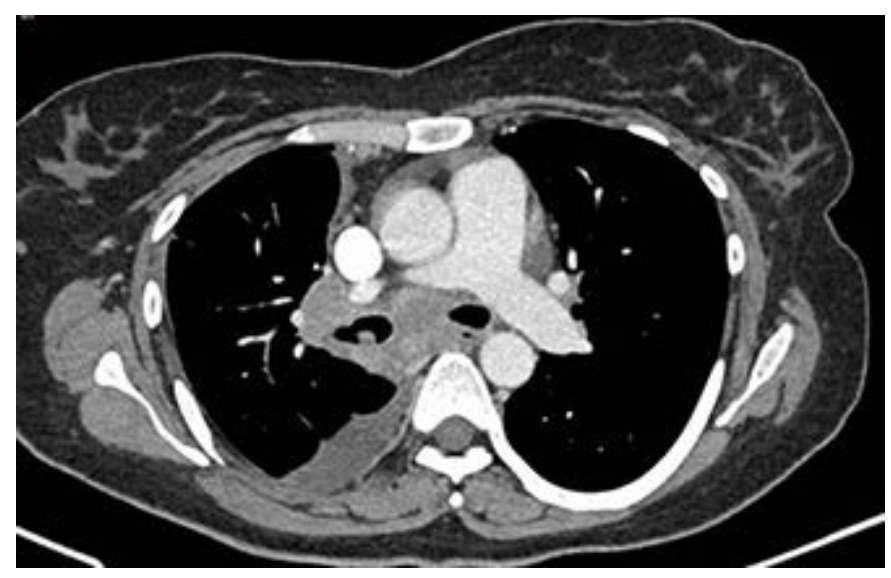

Figura 1. Tomografía de tórax en la que se observa lesión exofitica en el bronquio fuente derecho, la cual fue llevada a broncoscopia diagnostica.
Cuando se identifican lesiones bronquiales, el diagnóstico diferencial está en relación con la presencia de secreciones obstruyendo la luz, particularmente cuando se trata de lesiones ovoideas, en ángulo obtuso y con historia de tabaquismo, mientras la presencia de lesiones redondeadas, lobuladas, de márgenes poco definidos y con más de 21.7 unidades Hounsfield, sugieren fuertemente un verdadero tumor (6).

En un estudio observacional publicado en el 2016, realizado por H-J Kim y colaboradores, donde se estudiaron 53.036 imágenes de TAC de baja dosis, solo se identificaron nódulos en la vía aérea en el $0.6 \%$ de los pacientes con una alta correlación bronscocópica lo que sugiere que el seguimiento con TAC puede ahorrar estudios endoscópicos innecesarios en pacientes con alta sospecha de malignidad (7). En este estudio, además, fue común la identificación de lesiones transitorias por lo que en pacientes asintomáticos podría optarse por control imagenológico en un mes, en concordancia con la guía para el estudio de cáncer de pulmón de la National Comprehensive Cancer Network (NCCN) (7).

La implementación del TAC multidetector y la mayor disponibilidad de diferentes técnicas de broncoscopia han permitido mejorar el abordaje diagnóstico. La combinación de estas técnicas podría ayudar a revelar las ubicaciones y morfologías de los tumores, invasiones extramurales e implicaciones longitudinales de tumores, morfologías y extensión de la estenosis luminal, distancias entre el bronquio principal y la carina, así como características internas de los tumores. Cuando la lesión es focal sugiere más un tumor primario endobronquial $(1,8)$.

Los tumores endobronquiales difieren según la localización en el árbol bronquial y pueden sugerir algunas entidades clínicas como las siguientes.

1. Lesiones de tráquea: carcinoma escamo celular, carcinoma adenoide quístico, metástasis, condromas, papilomas, hamartomas y lipomas (8).

2. Lesiones bronquiales: tumor carcinoide, carcinoma mucoepidermoide, carcinoma escamo celular, hamartomas, metástasis (8).

3. Lesiones mixtas: siempre pensar en enfermedades que pueden ser sistémicas o que no son de origen neoplásico como policondritis recidivante, granulomatosis con poliangeitis, infecciones, papilomatosis, amiloidosis, traqueobroncopatía osteocondroplástica, metástasis, bronquiolitos, 
pseudotumor mucoide o cuerpos extraños (especialmente en niños) (8).

Otros estudios realizados con menor frecuencia incluyen el uso de resonancia magnética o tomografía por emisión de positrones, sin embargo, la primera puede ser útil para el estudio del carcinoma adenoide quístico o lesiones con atenuación grasa como hamartomas y lipomas (1), mientras la tomografía por emisión de positrones (PET) permite identificar la captación del 18-Flurodesoxiglucosa (FDG) que sugiere etiología maligna cuando esta se encuentra aumentada (1).

Posterior a la obtención de imágenes tomográficas, el estudio de elección corresponde a la broncoscopía flexible (Figuras 2 y 3), dado que permite la obtención de estudios histopatológicos. Para la obtención de muestras se puede hacer uso de fórceps, aguja fina o criobiopsia (tomada mediante crioterapia) $(9,10)$. El rendimiento diagnóstico mediante el uso de fórceps se encuentra entre el 72 a $82 \%$, por lo que se sugiere tomar al menos tres muestras, siempre que la condición lo permita, sin embargo, en el $10 \%$ de los casos no es posible. Cuando se combina el uso de la broncoscopia con punción de aguja fina del área comprometida, el rendimiento diagnóstico aumenta hasta $90 \%(9,10)$. En comparación, la broncoscopia rígida por sus ventajas adicionales en el apoyo de la ventilación, con canal de trabajo de mayor diámetro que permite mejor limpieza de secreciones o sangrado, instrumentación mayor o por la posibilidad de combinar broncoscopia flexible, puede utilizarse

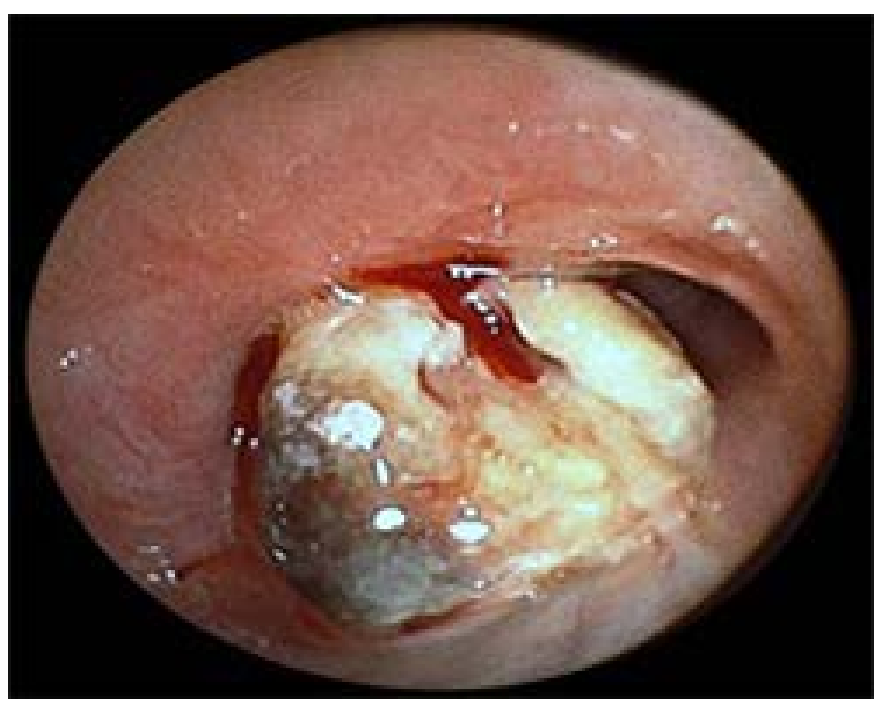

Figura 2. Sarcoma metastásico. Se puede ver la lesión endobronquial encontrada de la cual se toma biopsia. en escenarios particulares (2).

A su vez, la crioterapia ha ganado importancia en la actualidad, dado que es una opción tanto diagnóstica como terapéutica, con un rendimiento diagnóstico del $90-95 \%$ y al compararla con las técnicas de fórceps, permite obtener muestras de mejor calidad, menos hemorrágicas, de mayor tamaño y pueden tomarse un mayor número de muestras cuando es pertinente $(10,11)$.

El ultrasonido endobronquial (EBUS) puede ser útil para el estudio de extensión de las lesiones y su relación con estructuras del mediastino (1). Un estudio publicado en 2018 por Deng y colaboradores encontró una sensibilidad del 85-95\%, especificidad del $99 \%$ para estadiaje del mediastino en neoplasias pulmonares, pero su utilidad en lesiones benignas fue mucho menor de 55-67\% (12).

\section{Tratamiento}

Debe ser dirigido por un equipo multidisciplinario de neumólogos, radiólogos, cirujanos de tórax y anestesiólogos. El tratamiento de elección es endoscópico (Ver Tabla 1) y la elección de la técnica se realiza con base en las características morfológicas del tumor, el grado de obstrucción endoluminal, la presencia de compresión extrínseca o mixta, así como la disponibilidad y entrenamiento del médico (2).

Entre las opciones terapéuticas se encuentran:

1. Observación: se reserva para pacientes asintomáticos con lesiones que tengan

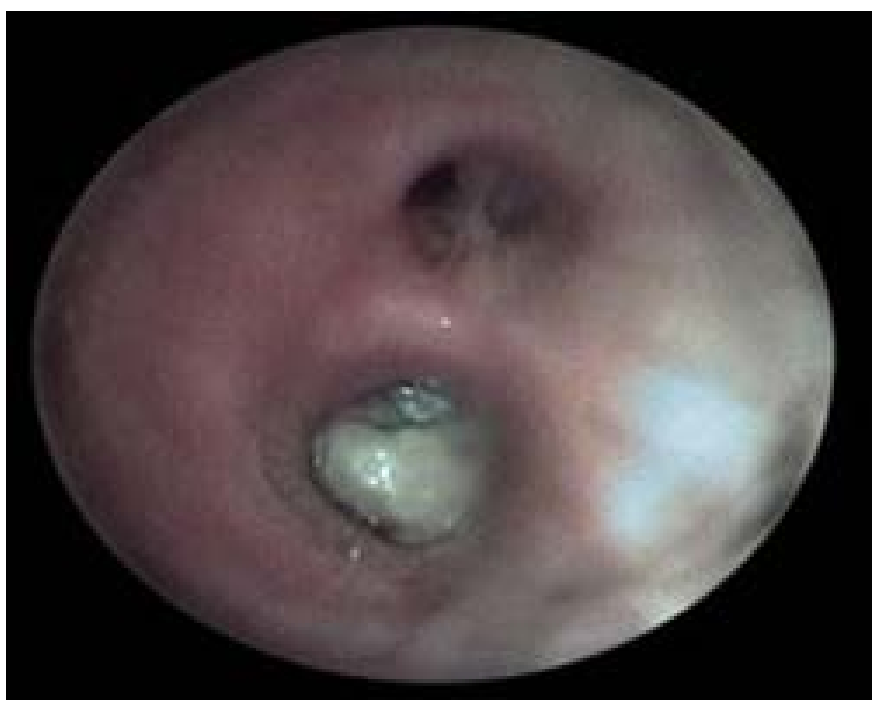

Figura 3. Melanoma maligno que obstruye luz bronquial. 
Tabla 1. Opciones de tratamiento endoscópico

\begin{tabular}{|c|c|c|c|}
\hline Técnica & Indicaciones & Ventajas & Desventajas \\
\hline Ablación con Laser & $\begin{array}{l}\text { Lesiones proximales con mínima } \\
\text { infiltración mucosa } \\
\text { Hemostasia } \\
\text { Granulomas }\end{array}$ & $\begin{array}{l}\text { Rápido } \\
\text { Inmediato } \\
\text { Efecto prolongado }\end{array}$ & $\begin{array}{l}\text { Riesgo de quemaduras, estenosis, edema, } \\
\text { perforación y hemorragia. } \\
\text { Alto costo } \\
\text { Complicaciones en }<5 \%\end{array}$ \\
\hline $\begin{array}{r}\text { Coagulación con argón } \\
\text { plasma }\end{array}$ & $\begin{array}{l}\text { Lesiones proximales especialmente } \\
\text { hemorrágicas } \\
\text { Granulomas }\end{array}$ & $\begin{array}{l}\text { Puede moldearse y doblarse } \\
\text { Menor riesgo de sangrado y } \\
\text { perforación }\end{array}$ & $\begin{array}{l}\text { Alto costo } \\
\text { Velocidad de respuesta moderada }\end{array}$ \\
\hline Microdesbridamiento & Lesiones proximales & $\begin{array}{l}\text { Rápido } \\
\text { No utiliza calor }\end{array}$ & $\begin{array}{l}\text { No alcanza lesiones distales } \\
\text { Riesgo de perforación } \\
\text { No se debe usar en lesiones vascularizadas }\end{array}$ \\
\hline Electrocauterio & $\begin{array}{l}\text { Displasia y carcinoma microinvasivos } \\
\text { Tumores exofíticos } \\
\text { Hemostasia } \\
\text { Granulomas }\end{array}$ & $\begin{array}{l}\text { Modificar el calor } \\
\text { Elección en áreas poco } \\
\text { vascularizadas } \\
\text { No produce cambios } \\
\text { histológicos } \\
\text { Respuesta rápida }\end{array}$ & $\begin{array}{l}\text { Desnaturaliza proteínas de vía aérea } \\
\text { No en lesiones de base ancha } \\
\text { Riesgo de quemadura, estenosis, perforación, } \\
\text { neumotórax y sangrados }\end{array}$ \\
\hline $\begin{array}{r}\text { Solidificación con } \\
\text { microondas }\end{array}$ & Tumores en ángulos agudos & $\begin{array}{l}\text { Se puede usar con } \\
\text { oxigenoterapia por poca } \\
\text { producción de humo }\end{array}$ & $\begin{array}{l}\text { No es útil en lesiones grandes por bajo poder } \\
\text { de cauterización }\end{array}$ \\
\hline Crioterapia & $\begin{array}{l}\text { Lesiones sin invasión al cartílago y poca } \\
\text { profundidad. } \\
\text { Granulomas }\end{array}$ & $\begin{array}{l}\text { Procedimiento rápido y fácil } \\
\text { Bajo costo } \\
\text { No requiere calor } \\
\text { Bajo riesgo de } \\
\text { complicaciones (principal: } \\
\text { sangrados) }\end{array}$ & $\begin{array}{l}\text { Puede requerir múltiples sesiones } \\
\text { Intubación y sedación en el procedimiento } \\
\text { Efecto puede ser rápido o retardado de } \\
\text { acuerdo con la técnica utilizada }\end{array}$ \\
\hline Terapia fotodinámica & $\begin{array}{l}\text { Lesiones de máximo } 6 \text { a } 7 \mathrm{~mm} \text { de } \\
\text { profundidad y menor de } 2 \mathrm{cms} \text { de } \\
\text { tamaño }\end{array}$ & Lesiones múltiples & $\begin{array}{l}\text { Alto costo } \\
\text { Riesgo de lesión por quemaduras } \\
\text { Fotosensibilidad } \\
\text { Imagen varía según el equipo utilizado } \\
\text { Mayores estudios en lesiones escamosas }\end{array}$ \\
\hline Braquiterapia & $\begin{array}{l}\text { Lesiones de poca profundidad } 3 \mathrm{~mm} \\
\text { Pacientes no candidatos a cirugía } \\
\text { Lesiones remanentes después de cirugía }\end{array}$ & $\begin{array}{l}\text { Lesiones ocultas por } \\
\text { radiologías } \\
\text { Sinergia con radioterapia }\end{array}$ & $\begin{array}{l}\text { Riesgo de hemoptisis masiva } \\
\text { Lesiones por radiación: bronquitis, necrosis, } \\
\text { fistulas, estenosis. }\end{array}$ \\
\hline Debulking mecánico & $\begin{array}{l}\text { Lesiones proximales con bajo riesgo de } \\
\text { sangrado }\end{array}$ & $\begin{array}{l}\text { Barata } \\
\text { Rápida }\end{array}$ & $\begin{array}{l}20 \% \text { de complicaciones serias: perforación y } \\
\text { hemorragia }\end{array}$ \\
\hline $\begin{array}{r}\text { Colocación de Stent de } \\
\text { silicona }\end{array}$ & $\begin{array}{l}\text { Obstrucción extrínseca o mixtas como } \\
\text { terapia paliativa }\end{array}$ & $\begin{array}{l}\text { Se retira fácilmente } \\
\text { Baja reacción isquémica o } \\
\text { granulomatosa }\end{array}$ & $\begin{array}{l}\text { Riesgos durante colocación } \\
\text { Altera el aclaramiento ciliar } \\
\text { Riesgo de migración }\end{array}$ \\
\hline $\begin{array}{r}\text { Colocación de Stent } \\
\text { metálico }\end{array}$ & $\begin{array}{l}\text { Segunda línea cuando otros métodos } \\
\text { fallan excepto en lesión necróticas, } \\
\text { fistulas o con mucha distorsión de la vía } \\
\text { aérea }\end{array}$ & $\begin{array}{l}\text { Fácil de colocar } \\
\text { Aclaramiento ciliar se } \\
\text { mantiene }\end{array}$ & $\begin{array}{l}\text { Difícil de remover } \\
\text { Riesgo de perforación } \\
\text { Reacción granulomatosa }\end{array}$ \\
\hline
\end{tabular}

Fuente: elaboración propia, adaptada de las referencias 2,9,13,14,15,16. 
características y probabilidades altas de ser benignas o de lesiones múltiples y/o grandes que no se beneficiarían de tratamientos más agresivos (9).

2. Técnicas endoscópicas: son la primera línea tratamiento para este tipo de lesiones. La broncoscopia flexible sirve tanto de diagnóstico como tratamiento, permitiendo la resección completa de lesiones en la primera sesión, cuando son pequeñas o pedunculadas $(9,13)$.

Entre las técnicas para resección de lesiones tumorales endobronquiales tenemos:

a- Ablación con láser: son pulsaciones cortas en el eje bronquial, que permiten permeabilizar la vía aérea con lesiones obstructivas críticas y de emergencia, siendo un método rápido y con efecto prolongado. Sus principales desventajas se relacionan con los efectos secundarios al calor y el alto costo de los equipos. Se utilizan actualmente diodos láser que con el paso de electrones en su interior forman el haz de luz en la dirección deseada, o el NdYAG, que posee un cristal de neodimio que genera la actividad láser bombeando ópticamente los diodos láser $(2,13,14)$.

b- Coagulación con argón plasma: método de electrocirugía sin contacto que utiliza el gas argón y energía eléctrica de alto voltaje y alta frecuencia, siendo muy utilizada en lesiones proximales y hemorrágicas (Figura 4). Los riesgos de complicaciones son menores que con láser; la velocidad de la respuesta es de moderada a rápida pero su costo sigue siendo elevado $(2,13,14)$.

c- Microdesbridamiento: es la resección de la lesión con un catéter rígido; es de bajo costo y por lo tanto fácil acceso, pero no sirve para lesiones distales ni vascularizadas, con riesgo importante de perforación (13).

d- Electrocauterio: destrucción térmica de los tejidos con una corriente eléctrica de alta frecuencia, que permite modificar el calor para aumentar la solidificación del tumor y facilitar su resección, con tasas de remisión de hasta el $91 \%$, obteniendo un efecto terapéutico rápido, pero puede desnaturalizar la pared de la vía aérea. Es de bajo costo, ampliamente disponible, no produce cambios histológicos, pero no es útil en lesiones de base ancha. Se utiliza actualmente para lesiones poco vascularizadas, displásicas o microinvasivas $(3,14)$.

e- Solidificación con microondas: desnaturalizar y solidificar las lesiones utilizando microondas es útil en tumores en ángulos agudos, y hay poca producción de humo durante el procedimiento, pero por su bajo poder de cauterización no es útil en lesiones grandes (13).

f- Crioterapia: es la técnica más utilizada actualmente para diagnóstico y tratamiento de lesiones bronquiales e incluso es ampliamente utilizada en lesiones parenquimatosas. Su uso requiere de sedación y potencialmente intubación orotraqueal. Consiste en la aplicación de temperatura de 70 grados bajo cero, durante $30 \mathrm{a}$ 60 segundos, provocando el efecto citotóxico del frío (deshidratación y cristalización) con ciclos de congelación rápida y descongelación lenta. Su uso se reserva en lesiones con una profundidad menor a $3 \mathrm{~mm}$ sin invasión cartilaginosa.

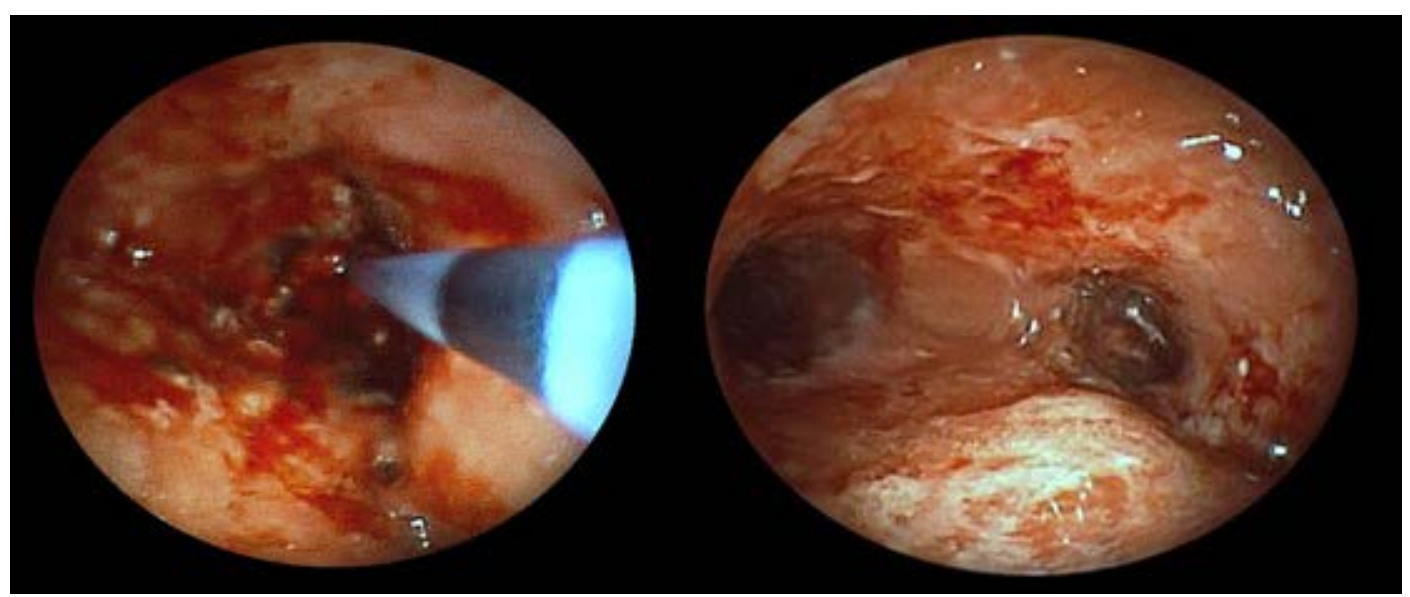

Figura 4. Carcicoma escamolecular: se lleva a terapia con argón plasma y se muestra recanalización de ambos bronquios. 
Esta técnica es de bajo costo y ampliamente disponible, con remisión histológica aproximada del $90 \%$, bajo riesgo de complicaciones (sangrado y neumotórax), pero puede requerir múltiples sesiones para una resección completa, con efecto más retardado en relación con otras técnicas $(13,14)$. En un estudio realizado por Chung y publicado en 2019, se evaluó el uso de crioterapia en el manejo de masas endobronquiales; se incluyó a 67 pacientes, concluyendo mayor mejoría de síntomas posterior al procedimiento, mejoría clínica sostenida y mejoría clínica previa a la quimioterapia (Odds Ratio de 1.3, 3.7 y 4.3 respectivamente). En este estudio los pacientes sometidos a crioterapia con quimioterapia tuvieron mayor sobrevida con respecto a solo el procedimiento (15).

g- Terapia fotodinámica: reacción fototóxica local usando ondas específicas de $630 \mathrm{~nm}$. Se puede utilizar en lesiones múltiples, principalmente en lesiones escamosas, en algunas lesiones exofíticas menores a $2 \mathrm{~cm}$ y lesiones de máximo 6 a $7 \mathrm{~mm}$ de profundidad. Permite la remisión en el $70 \%$ de los casos por lo cual es una técnica ampliamente estudiada, sin embargo, es de alto costo y existe el riesgo de lesión por quemadura y fotosensibilidad. La imagen varía según el equipo utilizado $(2,13,14)$.

h- Braquiterapia: aplicación de isótopo radioactivo de Iridio 192 a través de un catéter. Se usa en lesiones de poca profundidad $(3 \mathrm{~mm})$, no candidatos a cirugía por recurrencia, lesiones remanentes después de cirugía, lesiones ocultas por radiología y es útil en sinergia de radioterapia, con sobrevida de $92 \%$ a los 2 años. El riesgo de hemoptisis masiva es alto, además puede producir lesiones por radiación (necrosis, bronquitis, estenosis y fistulas) $(2,13,14)$.

i- Debulking mecánico: resección de tejidos usando tubos o fórceps rígidos, se usa en lesiones proximales que tienen bajo riesgo de sangrado. Es una técnica rápida y de bajo costo, pero con riesgo muy alto de complicaciones relacionadas con hemorragia (20\% de los pacientes) o perforación de la vía aérea (13).

j- Stent de silicona: colocación con fórceps o broncoscopia rígida; es una terapia paliativa para obstrucción extrínseca o mixta de la vía aérea. Es rápida, tiene buena tolerancia y se puede retirar fácilmente si es necesario. Tiene baja reacción granulomatosa o isquémica, sin embargo, presenta riesgo de migración con la compresión extrínseca y altera el aclaramiento mucociliar (13).

k- Stent Metálico: colocación mediante broncoscopia rígida o flexible; es un método de segunda línea, cuando otros han fallado y cuando no son lesiones necróticas, fistuladas o con distorsión de la vía aérea. Es de fácil colocación y no altera el aclaramiento ciliar, pero es difícil de retirar, produce reacción granulomatosa y tiene riesgo de perforación (13).

3. Intervenciones quirúrgicas: Se utiliza cuando el tratamiento endoscópico no es suficiente y se cumple con las siguientes indicaciones: a) biopsia no pudo excluir malignidad, b) bronquiectasias extensas, c) neumonía de organización o postobstructiva significativa, d) infiltración del tumor a la pared bronquial y, e) complicación de la vía aérea distal a la obstrucción (16).

En el cuidado posoperatorio es prioritario el manejo del dolor; requiere tos efectiva y buena terapia respiratoria y ante la sospecha o signos de atelectasia distal a la anastomosis bronquial, se debe realizar broncoscopia si la condición del paciente es adecuada (16). La principal complicación es la estenosis, requiriendo colocación de stent o reintervenciones, prefiriendo el tratamiento endoscópico cuando su aparición es tardía (16).

El pronóstico es variable, depende de la localización y extensión de la lesión, malignidad y comorbilidades.

A continuación, se hará una revisión de los tumores más frecuentes según su clasificación histopatológica, para el resto de los tipos histológicos (Ver Tabla 2).

\section{Tumores malignos}

1. Tumores carcinoides

Los tumores carcinoides de origen bronquial corresponden al $25 \%$ de los tumores carcinoides y a $1-2 \%$ de las neoplasias pulmonares. Tiene una incidencia de 0.2-2/100.000 habitantes por año en Estados Unidos y Europa $(1,8)$. Pueden corresponder al bronquio principal, lobar o segmentario (8). Son carcinomas de bajo, mediano y alto grado, con presentación clínica variable, muy vascularizados por lo cual el $50 \%$ de los pacientes manifiestan hemoptisis (17). De forma excepcional pueden producir síndromes paraneoplásicos, sin embargo, es menos común en comparación con los tumores intestinales, excepto cuando hay metástasis a otros 
Tabla 2. Histopatología de los tumores endobronquiales

\begin{tabular}{|c|c|}
\hline Benignos & Malignos \\
\hline 1. Tumores de origen mesenquimal & 1. Tumores de origen epitelial \\
\hline Hamartomas & Carcinoma escamo celular \\
\hline Lipomas & Adenocarcinoma \\
\hline Condromas & Carcinoma de célula pequeñas \\
\hline Leiomioma pulmonar primario & tumor neuroendocrino de células grandes \\
\hline Tumores de células granulares & Tumor carcinoide \\
\hline \multicolumn{2}{|l|}{ Tumores neurogénicos } \\
\hline $\begin{array}{l}\text { 2. Tumores que se originan de glándulas } \\
\text { submucosas }\end{array}$ & 2. Tumores que se originan de glándulas submucosas \\
\hline Adenoma de glándulas mucosas & Carcinoma mucoepidermoide \\
\hline \multirow[t]{3}{*}{ Adenoma pleomórfico } & Carcinoma adenoide quístico \\
\hline & Carcinoma epitelial -mioepitelial (EMEC) \\
\hline & Carcinoma de células acinares pulmonares \\
\hline 3. Tumores de origen epitelial & 3. Tumores de origen mesenquimal \\
\hline Papilomas & Sarcoma pulmonar primario \\
\hline Papiloma de células escamosas & $\begin{array}{l}\text { Metástasis restringidas al bronquio: mama, riñón, colon y } \\
\text { melanoma }\end{array}$ \\
\hline Papiloma de células columnares & 4. Tumores bifásicos malignos \\
\hline \multirow[t]{7}{*}{ Pólipos fibroepiteliales } & Carcinoma pleomórfico \\
\hline & Carcinosarcoma \\
\hline & Blastomas pulmonares \\
\hline & 5. Otros \\
\hline & Linfoma \\
\hline & Melanoma maligno \\
\hline & $\begin{array}{l}\text { Invasión directa de otro cáncer: tiroides, pulmón, laringe y } \\
\text { esófago. }\end{array}$ \\
\hline
\end{tabular}

Fuente: elaboración propia, adaptada de las referencias 1,2,16.

sitios como el hígado $(17,18)$.

No tiene predisposición por ningún sexo, con una media de 45 años para su aparición; los atípicos son más comunes a edades avanzadas, hay reportes de asociación con el tabaquismo, mientras los de origen bronquial son más comunes en los niños (17).

En la TAC se observa una masa esférica u ovalada de bordes lobulados y con aumento de la intensidad, muy vascularizada, captando contraste (con excepciones), con calcificaciones excéntricas en el $26 \%$ de los casos (1). Hasta el $85 \%$ de los casos pueden ser multifocales y con complicaciones en el parénquima distal $(1,19)$. También es típico encontrar una masa parenquimatosa que se extiende de forma adyacente y solo una pequeña lesión endoluminal, que se conoce como signo de punta de iceberg (19).
En la broncoscopia, se observan lesiones endobronquiales, caracterizadas por ser nódulos suaves, de aspecto liso, color rojo cereza y polipoides $(1,17)$.

El tratamiento de elección es el abordaje quirúrgico y es la única opción curativa, con una sobrevida posterior a la resección a los 5 y 10 años en los típicos mayor de $90 \%$ y los atípicos del 70 y $50 \%$, respectivamente (20). En las guías de National Comprehensive Cancer Network (NCCN) no hay lugar para terapias sistémicas adyuvantes, pero se puede considerar en los casos de falta de respuesta (20).

2. Carcinoma escamocelular (primario endobronquial)

Son tumores bronquiales muy raros, solo se 
encuentra en $0.19 \%$ de los pacientes, relacionados con el tabaquismo y con edades avanzadas, entre los 50 y 70 años, son más comunes en hombres, así como su variante pulmonar (1). Histológicamente son idénticos, pero es la segunda neoplasia pulmonar que afecta el árbol bronquial, siendo la tráquea su localización más común (8). Generalmente es asintomático hasta que ocluye el $50 \%$ de la luz bronquial y produce síntomas inespecíficos tos, disnea, hemoptisis y sibilancias $(1,8)$.

Endoscópicamente es una lesión polipoide, sésil y focal, con estenosis excéntrica o engrosamiento circunferencial de la tráquea distal, de bordes irregulares (Figura 5) y que puede invadir mediastino por extensión directa o diseminación linfática, que muestran alta captación con FDG-PET $(1,8)$.

La masa puede crecer desproporcionadamente causando atelectasias y neumonía posobstructiva $(1,21)$. En TAC se puede observar masa endoluminal, así como invasión a estructuras vecinas y cavitación hasta en el $10 \%$ de las lesiones, sin embargo, en algunos casos solo se identifica engrosamiento localizado de paredes bronquiales, lo que puede imitar lesiones benignas e inflamatorias, por lo que se sugiere seguimiento cercano y estudios de tamizaje en pacientes con alta sospecha o con antecedente de tabaquismo $(1,21)$.

\section{Tumores malignos de glándulas salivares}

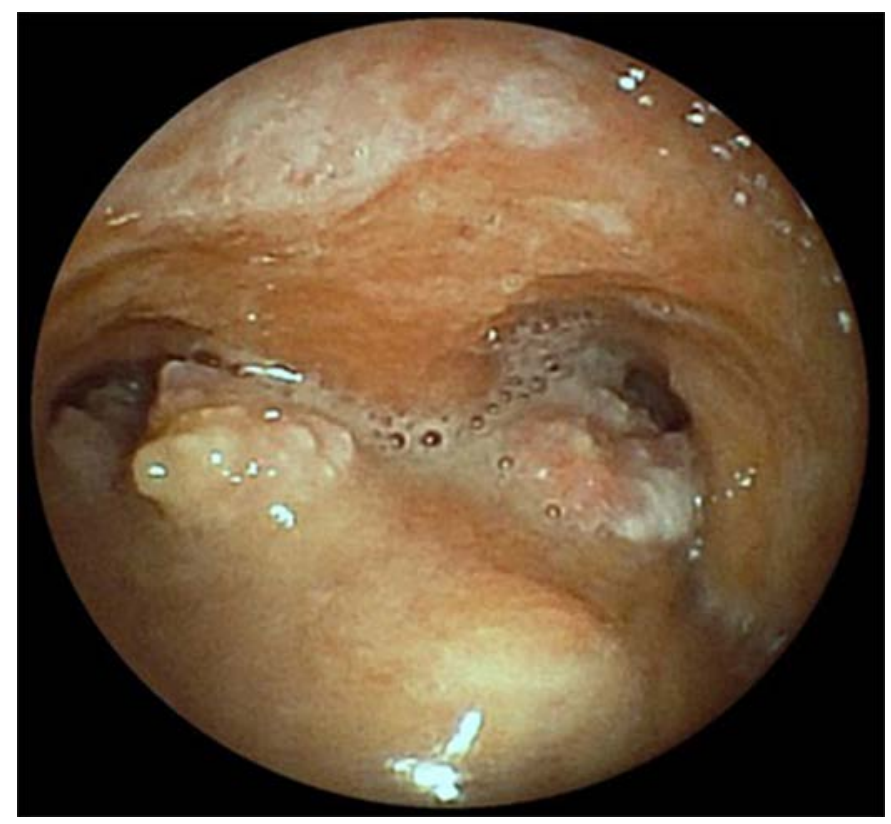

Figura 5. Carcinoma escamocelular que compromete carina y ambos bronquios fuentes.
Son muy raros, corresponden a menos del $2 \%$ de todas las neoplasias pulmonares; se presentan en pacientes jóvenes no fumadores, con una mayor predisposición en el sexo femenino. Tienen mejor pronóstico que otros tipos histológicos [1].

El carcinoma adenoide quístico es el más común de esta estirpe, con dos tercios de los casos, y es el segundo tumor más común de la vía aérea central con prevalencia de 0.04-0.2 \% (19).

Sus síntomas son inespecíficos ya sea por obstrucción o infecciones, lo que generalmente retrasa el diagnóstico, pero aun en estadios avanzados el pronóstico es bueno, excepto cuando hay compromiso metastásico, sin embargo, la recurrencia es común y tienen menor periodo libre de enfermedad comparado a otros tumores, particularmente cuando se localiza en la tráquea (22).

La TAC es el estudio de imagen de elección, permite identificar una masa de bordes lisos, que viene de la submucosa y que puede ocupar más de la mitad de la circunferencia de la tráquea, siendo común la extensión extraluminal $(1,19)$. Las adenomegalias mediastinales son raras, al igual que la metástasis; cuando estas se presentan son pulmonares, óseas y hepáticas (22). En broncoscopia encontramos una lesión con la mucosa intacta, se origina de la submucosa, tiene borde suave, con extensión longitudinal o circunferencial a través de la submucosa $(1,19)$.

El carcinoma mucoepidermoide es un tumor raro, se presenta en un tercio de los casos de esta estirpe, pero solo en $0.1-0.2 \%$ de las neoplasias pulmonares. No tiene relación con el tabaquismo, la edad media de presentación es de 40 años y sin predisposición según el sexo (1). Es más frecuente que se origine en un bronquio lobar antes que el principal o la tráquea. Puede ser de alto o bajo grado (1).

Es común que su identificación sea incidental ya que son comúnmente asintomáticos. Las imágenes tomográficas identifican una masa de bordes lisos, no esférica, lobulado con realce medio, que se adapta al bronquio, puede haber un bronquio distal dilatado, atelectasias e impactación de moco (1). Si son lesiones periféricas se observa un nódulo definido de bordes lisos menor de $2 \mathrm{~cm}$ con calcificaciones puntuales en la mitad de los pacientes (1). Se ha reportado captación variable con FDG-PET, por lo que en casos de alta captación es difícil diferenciar de adenocarcinomas. En broncoscopia se observa una masa rosada, exofítica intraluminal que puede ser sésil polipoide, vascularizada, con base ancha conectada al 
bronquio o pedunculada, que puede confundirse con un carcinoide (1).

Los otros tipos de tumores de glándulas salivares, solo se han reportado de forma aislada en la literatura y son entidades muy raras

\section{Tumores Benignos}

\section{Hamartomas}

Son tumores mixtos con tejido óseo, grasa, cartílago y musculo liso, con predominio de cartílago y grasa en su variante endobronquial. Tienden a ser muy sintomáticos, lo cual depende del grado de obstrucción, presentándose con sibilancias, disnea, fiebre y hemoptisis. Corresponden al $70 \%$ de los tumores benignos bronquiales, con incidencia entre $0.025-1.4 \%$, excepto la localización traqueal que es extremadamente rara con 20 casos reportados en adultos (1).

Hay más predisposición en las mujeres en la sexta década de la vida, aunque algunos trabajos refieren que ocurre en varones con una relación 2-4:1 y refieren cierta relación con el tabaquismo y con enfermedades inflamatorias del pulmón (1). En la TAC se observa una masa heterogénea, con atenuación grasa y calcificaciones en roseta de maíz, típico de estas lesiones (23). Pueden no encontrarse estos hallazgos, lo que dificulta el diagnóstico (23). En la broncoscopia se encuentra una masa polipoide o pedunculada, lisa de bordes amarillentos sin involucrar la submucosa (24).

2. Papiloma

Son tumores raros, corresponden al $0.38 \%$ de las neoplasias pulmonares, el 7-8 \% de las benignas. Se clasifican en tres categorías: papilomas múltiples, pólipos inflamatorios y papiloma solitario (1). Papilomas bronquiales múltiples, también llamados recurrentes, se presentan en forma bimodal en jóvenes o adultos después de la cuarta década de la vida, relacionados con infección de virus de papiloma humano (VPH) 6 y 11(1). En la broncoscopia se observan lesiones friables que pueden estar ulceradas, su localización más frecuente es la laringe, produciendo estridor y obstrucción de la vía aérea; son lesiones queratinizantes en la histología (9).

En cambio, los papilomas solitarios son más frecuentes en hombres de edad media, generalmente fumadores o asociados a infección por VPH 16 y 18 , con una prevalencia estimada de 3.95 pacientes por cada 100.000 habitantes $(9,24)$. Corresponden a nódulos polipoides que se originan en la tráquea, bronquio lobar o segmentario, se originan de la superficie mucosa con tamaño entre $0.7-2.5 \mathrm{~cm}$ de diámetro (1). En la broncoscopia se observan como lesiones exofiticas, friables, que pueden estar ulceradas (9).

En la TAC se evidencia masa endoluminal polipoide con atelectasia distal o neumonía obstructiva y atrapamiento aéreo (9). Cuando afectan el árbol bronquial distal pueden formar masas o nódulos que si crecen mucho se pueden cavitar (9).

Otras variantes que se encuentran son los glandulares, con menos de 30 casos reportados, la variante, mixta glandular y escamosa es un poco más frecuente (9).

\section{Conclusiones}

Los tumores endobronquiales son entidades poco frecuentes; su diagnóstico es retador dado los hallazgos poco específicos, presentándose con una clínica variable y que depende del grado de obstrucción, tanto en tumores benignos como malignos. Esto retrasa el diagnóstico, por lo cual se requiere un abordaje multidisciplinario para un adecuado diagnóstico y tratamiento.

La broncoscopia corresponde al estándar de diagnóstico y tratamiento, con una amplia variedad de técnicas disponibles. Actualmente no hay ninguna técnica que sea superior a otra, por lo que su elección depende de la disponibilidad de cada una y la experticia que tenga el neumólogo intervencionista para realizarlas, siendo las más utilizadas el electrocauterio, la coagulación con argón plasma, el láser y la crioablación. Se destaca el hecho de que la evidencia científica disponible es escasa, proveniente de estudios observacionales predominantemente reportes de casos o experiencias de centros especializados, por lo cual se deben realizar estudios con grandes poblaciones y con diseños experimentales que permitan tomar decisiones basadas en la evidencia.

\section{Conflicto de Interés}

No existe ningún conflicto de interés para la realización o publicación de este artículo por parte de los autores.

Todos los autores han contribuido con la concepción, diseño del trabajo, obtención, interpretación y análisis de datos, así como la redacción y revisión del documento. 


\section{Referencias}

1. Stevic R, Milenkovic B. Tracheobronchial tumors. J Thorac Dis 2016;8(11):3401-3413. doi: 10.21037/jtd.2016.11.24

2. Gasparini S, Bonifazi M. Management of endobronchial tumors. Current Opinion in Pulmonary Medicine 2016;22(3):245-251. Disponible en: https://doi.org/10.1097/ MCP.0000000000000259

3. Junco BMD, Barreto BA, Castro GN, et al. Síndrome del lóbulo medio: presentación de un caso. Rev Arch Med Camagüey. 2017;21(6):2017. Disponible en: https://www. medigraphic.com/cgi-bin/new/resumen. cgi?IDARTICULO $=78854$

4. Hadda V, Khilnani GC. Lipoid pneumonia: an overview. Expert review of respiratory medicine 2010 Dec;4(6):799-807. doi: https://doi. org/10.1586/ers.10.74

5. Graham BL, Steenbruggen I, Miller MR, Barjaktarevic IZ, Cooper BG, Hall GL, et al. Standardization of Spirometry 2019 Update. An Official American Thoracic Society and European Respiratory Society Technical Statement. Am J Respir Crit Care Med 2019;200(8):e70-e88. doi: https://doi.org/10.1164/rccm.201908-1590ST

6. Hong SR, Lee YJ, Hong YJ, Hur J, Kim YJ, Choi BW, et al. Differentiation between mucus secretion and endoluminal tumors in the airway: analysis and comparison of CT findings. AJR Am J Roentgenol 2014 May;202(5):982-988. doi: 10.2214/AJR.13.11392

7. Kim H, Kim DK, Kim YW, Lee YJ, Park JS, Cho Y, et al. Outcome of incidentally detected airway nodules. Eur Respir J. 2016;47(5):1510. doi: 10.1183/13993003.01992-2015

8. Ngo AH, Walker CM, Chung JH, Takasugi JE, Stern EJ, Kanne JP, et al. Tumors and tumorlike conditions of the large airways. AJR Am J Roentgenol. 2013 Aug;201(2):301-313. doi: 10.2214/AJR.12.9043

9. Agarwal A, Agrawal A, Alagusundaramoorthy SS, Meena N. Benign Endobronchial Neoplasms: A Review. Pulmonary \& Respiratory Medicine 2015;5:1-7. doi:10.4172/2161-105X.1000275

10. Dooms C. Should we abandon standard forceps biopsy to diagnose an endobronchial lesion? The European RespiratoryJournal2012Mar;39(3):513514. doi: 10.1183/09031936.00150511
11. Fang Y, Hsieh M, Wang T, Lin H, Yu C, Chou C, et al. Removal of Endobronchial Malignant Mass by Cryotherapy Improved Performance Status to Receive Chemotherapy. The Scientific World Journal. 2014;ID369739. doi: https://doi. org/10.1155/2014/369739

12. Deng C, Dai F, Qian K, Tan Q, Wang R, Deng B, et al. Clinical updates of approaches for biopsy of pulmonary lesions based on systematic review. BMC Pulmonary Medicine 2018 Sep;18(1):146. doi: 10.1186/s12890-018-0713-6

13. Guibert N, Mhanna L, Droneau S, Plat G, Didier A, Mazieres J, et al. Techniques of endoscopic airway tumor treatment. Journal of Thoracic Disease. 2016 Nov;8(11):3343-3360. doi: 10.21037/jtd.2016.11.49

14. Aboudara, M., Rickman, O., \& Maldonado, F. Therapeutic Bronchoscopic Techniques Available to the Pulmonologist: Emerging Therapies in the Treatment of Peripheral Lung Lesions and Endobronchial Tumors. Clinics in Chest Medicine. 2020;41(1):145-160). doi: https://doi.org/10.1016/j.ccm.2019.11.003

15. Chung F, Chou C, Lo Y, Kuo C, Wang T, Wang $\mathrm{C}$, et al. Factors affecting survival in patients with endobronchial malignant mass after flexible Bronchoscopic cryotherapy: a cohort study. BMC Pulm Med. 2019;19(101):1-6. doi: 10.1186/ s12890-019-0854-2

16. Li Z, Zarogoulidis P, Kougioumtzi I, Darwiche K, Tsakiridis K, Katsikogiannis N, et al. Surgical approaches of endobronchial neoplasms. Journal of Thoracic Disease 2013;5 Suppl 4:S378-S382. doi: 10.3978/j.issn.2072-1439.2013.06.22

17. Agasarova A, Harnett C, Mulligan N, Majeed MS, Caimo A, Tamagno G. Management and Follow-up of Patients with a Bronchial Neuroendocrine Tumor in the Last Twenty Years in Ireland: Expected Inconsistencies and Unexpected Discoveries. International Journal of Endocrinology. 2018;ID1043287:1-8. doi: https://doi.org/10.1155/2018/1043287

18. Silva, C. Cárcamo Flores, M. C. Álvarez Sanz, C. Izquierdo Sancho, S. Novo Aparicio, M. Hernández García, M. Rascón Risco. Thoracic calcifications: beyond granulomatous disease. Review and keys for differential diagnosis. Póster ECR 2015 / C-2005. doi: 10.1594/ ecr2015/C-2005

19. Queiroz RM, Santana DBF, Nastri Filho R, Landell GAM, Félix PR, Valentin MVN. 
Endobronchial carcinoid tumor: Radiological findings of a clinical case. Rev Assoc Med Bras. 2018 Jan;64(1):15-18. doi: https://doi. org/10.1590/1806-9282.64.01.15

20. Hendifar AE, Marchevsky AM, Tuli R. Neuroendocrine Tumors of the Lung: Current Challenges and Advances in the Diagnosis and Management of Well-Differentiated Disease. Journal of thoracic oncology 2017 Mar;12(3):425436. doi: 10.1016/j.jtho.2016.11.2222

21. Song Y, Choi YW, Paik SS, Han DH, Lee KY. Endobronchial squamous cell carcinoma presenting as localized, long, continuous bronchial thickening on CT. Eur J Radiol. 2017 Jun;91:99-105. doi: 10.1016/j.ejrad.2017.04.003

22. Nuñez C V, Herrero C J, Artazkoz T JJ, Maeso F MC. Carcinoma adenoideo quístico pulmonar primario con metástasis precoces exclusivas hepáticas, un caso inusual. Revista de otorrinolaringología y cirugía de cabeza y cuello. 2018;78:413-6. doi: http://dx.doi.org/10.4067/ s0717-75262018000400413

23. Gargantilla Madera P, Montero Jiménez J, Belén Cuenca Abarca Pedro Avelino Pérez García A. Hamartoma endobronquial como causa de neumonía. Rev Clin Med Fam. 2018 Feb [citado 2021 Dic 13] ; 11( 1 ): 37-39. Disponible en: https://scielo.isciii.es / scielo.php? script $=$ sci_ arttext\&pid=S1699-695X2018000100037

24. Dalar L, Ozdemir C, Sokucu SN, Nur Urer H, Altin S. Bronchoscopic Treatment of Benign Endoluminal Lung Tumors. Canadian Respiratory Journal 2019;2019:5269728. doi: https://doi.org/10.1155/2019/5269728 